\title{
Genes frecuentemente asociados con muerte súbita en miocardiopatía hipertrófica primaria
}

\author{
Genes frequently associated with sudden death in primary hypertrophic cardiomyopathy
}

Diana L. Herrera-Rodríguez ${ }^{1 \neq}$, Armando Totomoch-Serra ${ }^{2,3 \ddagger}$, Sandra Rosas-Madrigal4, Claudia Luna-Limón ${ }^{5}$, Daniel Marroquín-Ramírez ${ }^{5}$, Alessandra Carnevale ${ }^{6}$, Rigoberto Rosendo-Gutiérrez ${ }^{6}$, María T. Villarreal-Molina ${ }^{4}$ y Manlio F. Márquez-Murillo ${ }^{5 *}$

${ }^{1}$ Servicios de Salud de Chihuahua, Secretaría de Salud, Chihuahua; ${ }^{2}$ Departamento de Genética y Biología Molecular, Centro de Investigación y Estudios Avanzados del Instituto Politécnico Nacional (IPN), Ciudad de México, México; ${ }^{3}$ Programa de Doctorado en Ciencias Médicas, Universidad de la Frontera, Temuco, Chile; ${ }^{4}$ Laboratorio de Enfermedades Cardiovasculares, Instituto Nacional de Medicina Genómica; ${ }^{5}$ Departamento de Electrocardiología, Instituto Nacional de Cardiología; ${ }^{6}$ Laboratorio de Enfermedades Mendelianas, Instituto Nacional de Medicina Genómica, Ciudad de México, México

‡La contribución de los autores fue equitativa y el orden de estos es arbitrario.

\section{Resumen}

La miocardiopatía hipertrófica (MCH) es el aumento de grosor de la pared ventricular izquierda no relacionada con otras alteraciones cardíacas. Es una enfermedad que puede presentar como primera manifestación clínica la muerte súbita y de ahí su relevancia clínica. Aunque se presenta sobre todo en la edad adulta, puede aparecer durante la infancia y adolescencia, en las que predominan los casos de origen hereditario. La MCH primaria, de causa genética, muestra en particular un patrón de herencia autosómico dominante en los 25 subtipos reconocidos en OMIM (Online Mendelian Inheritance in Man). Las proteínas codificadas por los genes mutantes forman parte del sarcómero en células musculares cardíacas, y las variantes patogénicas de filamentos gruesos son las de mayor frecuencia y peor pronóstico. En este artículo se describen la herencia mendeliana de la enfermedad y la relación con muerte súbita de los genes más frecuentemente encontrados en ella: MYBPC3 y MYH7.

Palabras clave: Miocardiopatía hipertrófica. Genes. MYBPC3. MYH7. Variantes genéticas. Muerte súbita.

\begin{abstract}
Hypertrophic cardiomyopathy is characterized by left ventricular hypertrophy without apparent cardiac justification. Sudden cardiac death may be the first manifestation of the disease. It occurs mainly in adulthood and can be seen in childhood and adolescence where genetic origin predominates. Primary HCM ("familial") is inherited in an autosomal dominant pattern in the 25 subtypes informed in Online Mendelian Inheritance in Man. The proteins encoded by the mutated genes are part of the sarcomere in the cardiac cells, being the thick filament the most frequently affected, with the worst prognosis. In the present article, we describe the Mendelian inheritance of the disease and the two most associated genes with sudden death: MYBPC3 and MYH7.
\end{abstract}

Key words: Hypertrophic cardiomyopathy. Genes. Gene variants. MYBPC3. MYH7. Sudden cardiac death.

Correspondencia:

*Manlio F. Márquez

E-mail: manlio.marquez@gmail.com
Disponible en internet: $30-01-2020$ Arch Cardiol Mex. 2020;90(1):58-68 www.archivoscardiologia.com 2604-7063/@ 2019 Instituto Nacional de Cardiología Ignacio Chávez. Publicado por Permanyer. Este es un artículo open access bajo la licencia CC BY-NC-ND (http://creativecommons.org/licenses/by-nc-nd/4.0/). 


\section{Introducción}

La miocardiopatía hipertrófica (MCH) es una enfermedad cardíaca que se caracteriza por el aumento del grosor de la pared del ventrículo izquierdo sin que exista una sobrecarga de volumen sanguíneo 0 alguna otra enfermedad cardíaca que explique dicha hipertrofia. ${ }^{1}$

A lo largo de la historia se la ha conocido con diferentes nombres, entre ellos "hipertrofia ventricular hereditaria", "cardiomiopatía hipertrófica hereditaria", "cardiomiopatía hipertrófica familiar", "hipertrofia septal asimétrica" y "estenosis subaórtica idiopática". Para este trabajo se utiliza el término "miocardiopatía", y no "cardiomiopatía", por dos razones: a) "miocardiopatía" define la entidad, una enfermedad del miocardio, y b) el término "cardiomiopatía" es un anglicismo, un calco del inglés cardiomyopathy.

La European Society of Cardiology clasifica la $\mathrm{MCH}$ en dos grupos: a) $\mathrm{MCH}$ "primaria" o de origen genético causada por mutaciones en diversos genes ${ }^{1}$ y b) $\mathrm{MCH}$ "secundaria" consecutiva a otra enfermedad, como hipertensión arterial sistémica, enfermedad valvular, o diversos síndromes metabólicos, como las enfermedades de Pompe, Fabry y Danon, que afectan la estructura del miocardiocito. Este trabajo se enfoca en la $\mathrm{MCH}$ primaria.

La MCH primaria se considera la enfermedad cardíaca de origen hereditario más frecuente, con una prevalencia de 1 en 500 individuos, de acuerdo con datos estadísticos obtenidos del estudio CARDIA (Coronary Artery Risk Development in Young Adults) $)^{2}$, y es la sexta causa más frecuente de muerte súbita en niños y adultos jóvenes en países desarrollados, según un estudio realizado en Australia y Nueva Zelanda ${ }^{3}$.

Los signos y síntomas se presentan en la tercera y cuarta décadas de la vida, con una amplia variación en el inicio y gravedad de las manifestaciones clínicas, desde individuos asintomáticos hasta la presencia de síncope, taquiarritmias o inicio con muerte súbita. Sin embargo, el diagnóstico de la enfermedad se establece por cambios morfológicos como el engrosamiento de la pared ventricular $\geq 13 \mathrm{~mm}$ (medido mediante ecocardiografía), con o sin obstrucción del tracto de salida del ventrículo izquierdo ${ }^{4}$, y cambios electrocardiográficos en la repolarización, inversión de la onda T en las derivaciones cardíacas $\mathrm{DI}$ y aVL, además de prolongación de los intervalos $\mathrm{QT}^{5}$, entre otros indicadores que reflejan la progresión del daño y el riesgo de muerte súbita cardíaca; este último es más alto en el grupo de edad de 14 a 35 años que tiene síntomas secundarios a la $\mathrm{MCH}^{6}$.

\section{Herencia mendeliana en $\mathrm{MCH}$}

Aunque se trata de una enfermedad con patrón de transmisión bien identificado, la MCH muestra una gran variabilidad clínica, con penetrancia dependiente de la edad, es decir, que el curso clínico tiene una gran heterogeneidad intrafamiliar e interfamiliar debido a factores modificadores que diversifican el fenotipo de cada paciente. Esta enfermedad es un buen ejemplo de "naturaleza versus crianza" ("nature versus nurture")

Diversos estudios genéticos y genómicos han tratado de dilucidar las causas de esta anomalía hereditaria y han identificado modificaciones en el DNA conocidas como variantes genéticas, las cuales pueden ser benignas, probablemente benignas, de significado incierto, probablemente patogénicas o patogénicas y pueden ocasionar cambios estructurales en las proteínas sarcoméricas del músculo cardíaco, lo que favorece la hipertrofia de los ventrículos, de manera predominante del ventrículo izquierdo.

En general, la $\mathrm{MCH}$ primaria tiene un patrón de herencia mendeliano del tipo autosómico dominante, esto es, que los hijos de un afectado poseen $50 \%$ de riesgo de heredar el alelo mutado y por tanto padecerán la enfermedad. No obstante, se conocen casos de novo que no tienen antecedentes familiares y aun así el afectado muestra una variante patogénica identificada, la cual heredará con la misma probabilidad $(50 \%)$ a su descendencia8; por otro lado, existen informes de casos que presentan mutaciones en los genes MYL3, MYH7 y MYBPC3 que sugieren un patrón de herencia autosómica recesiva, lo cual debe considerarse al momento de realizar la historia clínica. Es por ello que algunas investigaciones han tratado de dilucidar el efecto funcional de las variantes genéticas que dan origen a las alteraciones cardíacas, con el objetivo de facilitar el diagnóstico y ofrecer tratamiento temprano, incluso en pacientes asintomáticos. Por el momento, la identificación de las variantes causales, y su efecto funcional, ayuda a comprender el mecanismo que genera la hipertrofia y a predecir la gravedad del daño.

Gracias al uso de la secuenciación de nueva generación (SNG), el número de genes relacionados con la $\mathrm{MCH}$ se ha incrementado de forma notable en los últimos años ${ }^{9-11}$, con más de 50 genes publicados y cerca de 8,000 variantes (Tabla 1) en la base de datos "PubMed" relacionados con esta enfermedad, de los cuales sólo 25 son consistentes con el compendio de genes Online Mendelian Inheritance in Man (OMIM; http: www. omim.org), en el que se describen las características 
Tabla 1. Genes y tipo de variantes relacionadas con miocardiopatía primaria

\begin{tabular}{|c|c|c|c|c|c|c|}
\hline \multirow[t]{2}{*}{ Gen } & \multirow[t]{2}{*}{ Тіро МСH } & \multirow[t]{2}{*}{ Frecuencia } & \multirow[t]{2}{*}{ Sin inf } & \multicolumn{3}{|c|}{ Tipo de variante descrita (OMIM)* } \\
\hline & & & & BEN & PAT & INC \\
\hline ACTC $1^{12-17}$ & $11^{\mathrm{a}}$ & Rara $<1 \%$ & 29 & 8 & 29 & 30 \\
\hline$T N N C 1^{18}$ & $13^{\mathrm{a}}$ & Rara $<1 \%$ & 17 & 4 & 7 & 8 \\
\hline TNNI3 $3^{13,14,19-24}$ & $7^{\mathrm{a}}$ & Rara $<5 \%$ & 47 & 7 & 73 & 39 \\
\hline TNNT2 $^{13,14,19,21,22,25-27}$ & $2^{\mathrm{a}}$ & Rara $<5 \%$ & 65 & 11 & 81 & 53 \\
\hline TPM113,14,26,28,29 & $3^{\mathrm{a}}$ & Rara $<5 \%$ & 83 & 10 & 29 & 51 \\
\hline MYBPC $3^{13,14,17,21,22,26,30,31-55}$ & $4^{b}$ & $15-25 \%$ & 241 & 42 & 1014 & 302 \\
\hline$M Y H 6^{23,26,56}$ & $14^{\mathrm{b}}$ & Rara $<1 \%$ & 183 & 7 & 3 & 13 \\
\hline$M Y H 7^{13,14,17,21-23,30-32,49-52,55,57-69}$ & $1^{b}$ & $15-25 \%$ & 300 & 41 & 872 & 317 \\
\hline MYL2 $13,17,29,70-72$ & $10^{\mathrm{b}}$ & Rara $<2 \%$ & 37 & 18 & 25 & 24 \\
\hline$M Y L 3^{13,14,69,73-75}$ & $8^{b}$ & Rara $<1 \%$ & 21 & 5 & 30 & 21 \\
\hline ACTN2 $2^{13-15,76,77}$ & $23^{c}$ & Rara $<1 \%$ & 76 & 30 & 9 & 45 \\
\hline CSRP3 $3^{78,79}$ & $12^{c}$ & Rara < $1 \%$ & 24 & 9 & 5 & 12 \\
\hline$\angle D B 3^{80}$ & $24^{c}$ & Rara 1-5\% & 151 & 8 & 0 & 5 \\
\hline MYOZ267,81 & $16^{c}$ & Rara $<1 \%$ & 36 & 1 & 8 & 2 \\
\hline$M Y P N^{\beta 2}$ & $22^{c}$ & Rara $<5 \%$ & 42 & 39 & 4 & 30 \\
\hline$N E X N^{\beta 3}$ & $20^{c}$ & Rara $<1 \%$ & 35 & 10 & 4 & 45 \\
\hline TCAP 84 & $25^{c}$ & Rara $<1 \%$ & 31 & 1 & 0 & 4 \\
\hline $\operatorname{TTN}^{15,26,51,72,85}$ & $9^{c}$ & Rara $<5 \%$ & 3949 & 2 & 12 & 20 \\
\hline$V C L^{86}$ & $15^{c}$ & Rara $<1 \%$ & 54 & 24 & 3 & 30 \\
\hline CALR $3^{37}$ & $19^{d}$ & Rara $<5 \%$ & 4 & 0 & 0 & 1 \\
\hline$J P H 2^{88,89}$ & $17^{\mathrm{d}}$ & Rara $<1 \%$ & 6 & 13 & 6 & 15 \\
\hline$P L N^{\rho 0}$ & $18^{d}$ & Rara $<1 \%$ & 7 & 0 & 5 & 5 \\
\hline $\operatorname{CAV}^{91}$ & $1^{e}$ & Rara $<5 \%$ & 54 & 11 & 5 & 8 \\
\hline$M Y L K 2^{92}$ & $1^{e}$ & Rara < $5 \%$ & 39 & 17 & 3 & 7 \\
\hline PRKAG293-98 & $6^{e}$ & Rara $1 \%$ & 100 & 1 & 31 & 4 \\
\hline
\end{tabular}

* Información obtenida de PubMed hasta abril del 2018.

OMIM: Online Mendelian Inheritance in Man; Sin inf: sin información en las publicaciones médicas; BEN: benigna; PAT: patogénica; INC: incierta; a: proteína de filamentos delgados; b: proteína de filamentos gruesos; c: proteína de discos Z; d: proteínas incluidas en el manejo de Ca+; e: otras proteínas relacionadas.

clínicas, los genes con las variantes patogénicas más comunes identificadas y las consecuencias fisiopatológicas de estos cambios moleculares.

\section{Estructura de las células cardíacas y proteínas que subyacen a la $\mathrm{MCH}$}

El miocardiocito se encuentra constituido por cinco componentes: a) túbulos y sarcolema, b) retículo sarcoplásmico, c) elementos contráctiles, d) mitocondrias y e) núcleo. La unidad contráctil se conoce como sarcómero, el cual está formado por filamentos gruesos y delgados cuyas proteínas más importantes son miosina y actina (Fig. 1). Los filamentos gruesos están formados por cadenas pesadas de la proteína $\beta$-miosina. Ésta es una proteína hexamérica compuesta por dos cadenas ligeras esenciales, codificadas por el gen MYL2, dos cadenas ligeras regulatorias, codificadas 


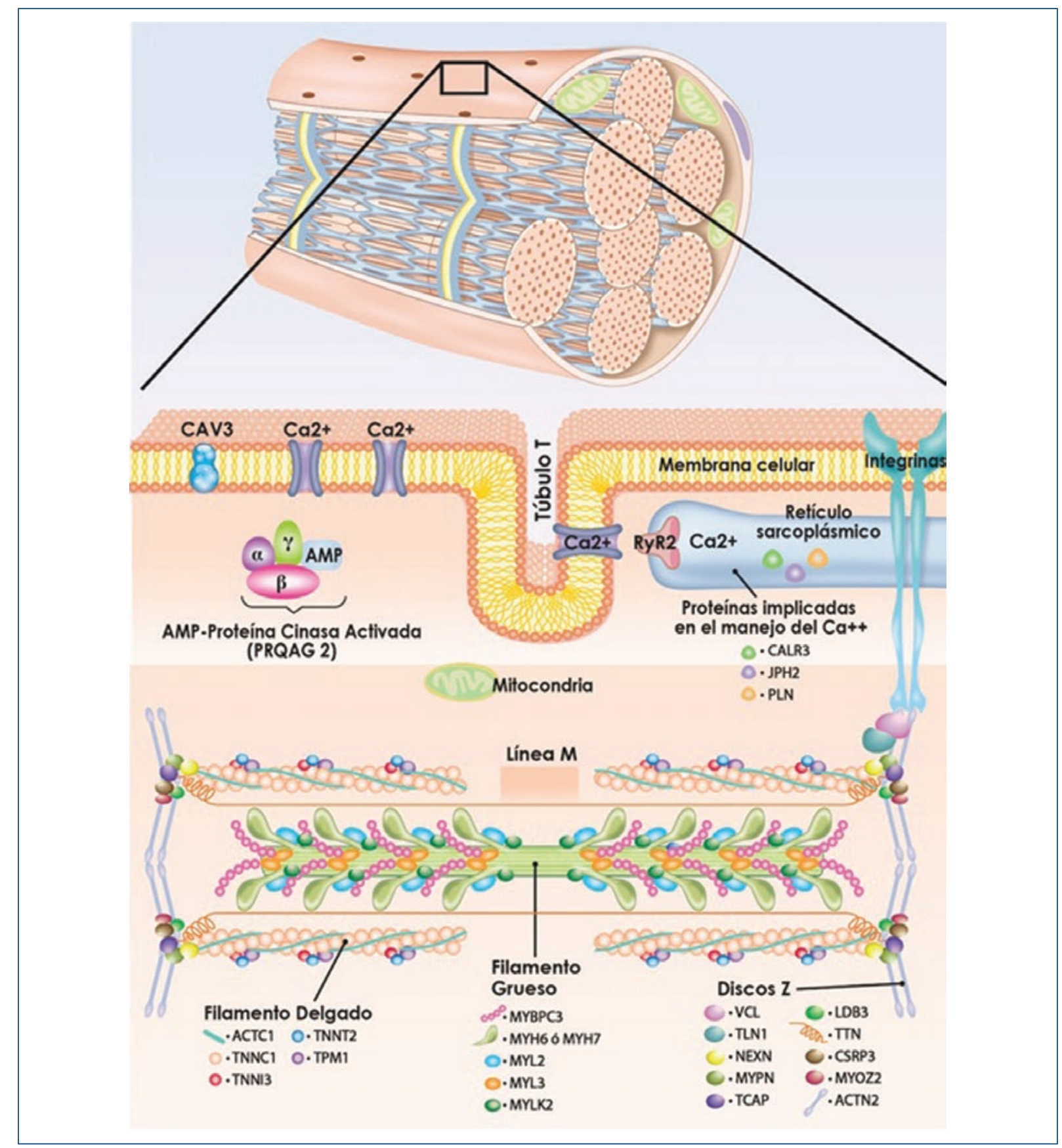

Figura 1. Sarcómero del músculo esquelético cardíaco. Se muestran las proteínas ubicadas en el sarcómero del músculo cardíaco y cuyas variantes patogénicas se relacionan con miocardiopatía hipertrófica primaria.

por el gen MYL3, y dos cadenas pesadas codificada por el gen $M Y H 7$, así como proteínas $\mathrm{C}$ de unión a miosina (gen MYBPC3). Los filamentos delgados están formados por actina (genes ACTC1 y ACTA1), el complejo de troponina (genes TNNC1, TNNI3, TNNT2) y tropomiosina (codificada por el gen TPM1) (Fig. 1). La alteración de uno o varios componentes del miocardiocito tiene repercusiones directas o indirectas en el desarrollo de la $\mathrm{MCH}$ primaria ${ }^{7,8}$.

\section{Alteraciones en miofilamentos gruesos y delgados}

Algunos estudios han demostrado que las variantes patogénicas en genes que codifican a proteínas de 
Tabla 2. Los genes del sarcómero cuyas variantes patogénicas o probablemente patogénicas se relacionan con $\mathrm{MCH}$ y muerte súbita

\begin{tabular}{|c|c|c|c|c|c|}
\hline Gen & Locus & Mim & Proteína & Herencia & HFMS \\
\hline \multicolumn{6}{|c|}{ Genes que codifican a proteínas de filamentos delgados } \\
\hline TNNC1 & $3 p 21.1$ & 613243 & Troponina C tipo 1 & $A D$ & $\mathrm{Sí}^{18}$ \\
\hline TNNI3 & $19 q 13.42$ & 613690 & Troponina I tipo 3 & $A D$ & Sín 19,20 \\
\hline TNNT2 & $1 q 32.1$ & 115195 & Troponina T tipo 2 & $A D$ & $\mathrm{Si}^{25}$ \\
\hline TPM1 & $15 q 22.2$ & 115196 & Tropomiosina 1 & $A D$ & $\mathrm{Si}^{28,99}$ \\
\hline ACTC1 & $15 q 14$ & 612098 & Actina alfa del músculo cardíaco 1 & $A D$ & $\mathrm{Si}^{12}$ \\
\hline \multicolumn{6}{|c|}{ Genes que codifican a proteínas de filamentos gruesos } \\
\hline MYBPC3 & $11 \mathrm{p} 11.2$ & 115197 & $\begin{array}{l}\text { Proteína C cardíaca de unión a } \\
\text { miosina }\end{array}$ & $\mathrm{AD} / \mathrm{AR}$ & 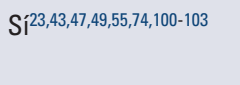 \\
\hline MYH6 & $14 q 11.2$ & 613251 & Cadena pesada de miosina alfa & $A D$ & Si ${ }^{56}$ \\
\hline MYH7 & $14 q 11.2$ & & Cadena pesada de miosina beta & $\mathrm{AD} / \mathrm{AR}$ & $\mathrm{Sí}^{46,49,55,57,58,67,100,104,105}$ \\
\hline MYL2 & $12 q 24.11$ & 608758 & $\begin{array}{l}\text { Cadena ligera de miosina } \\
\text { regulatoria ventricular }\end{array}$ & $A D$ & Sín 70,71 \\
\hline MYL3 & $3 p 21.31$ & 608751 & Cadena ligera de miosina 3 & $A D / A R$ & $S i^{36,73-75,106}$ \\
\hline
\end{tabular}

MIM: número correspondiente en la base de datos Mendelian Inheritance in Man; HFMS: historia familiar de muerte súbita; AD: autosómica dominante; AR: autosómico recesivo.

filamentos gruesos y delgados conducen a un incremento del gasto energético debido a la alteración en el uso y metabolismo del ATP9,10. Este "compromiso energético" podría resultar en una activación del sistema neuroendocrino con el fin de desarrollar una hipertrofia cardíaca compensatoria. Para estudiar los efectos funcionales de las variantes patogénicas, Coppini, et al. ${ }^{11}$ realizaron un estudio prospectivo durante 4.5 años, en el cual dividieron a un grupo de individuos afectados según la estructura alterada, y se conformaron así dos grupos: uno en el que los pacientes $(n=80)$ portaban variantes patogénicas de filamentos delgados y otro $(n=150)$ con variantes patogénicas de los filamentos gruesos, y encontraron que los pacientes con alteraciones de filamentos delgados mostraban una mayor disfunción ventricular izquierda e insuficiencia cardíaca, en comparación con el grupo de alteraciones en los filamentos gruesos, que presentaron mayor fibrosis, menor hipertrofia con distribución atípica, disfunción diastólica grave, respuesta anormal de la presión arterial durante el ejercicio y antecedente familiar de muerte súbita cardíaca. Por otra parte, los individuos con variantes patogénicas que afectan a filamentos gruesos sufrieron mayor obstrucción del tracto de salida del ventrículo izquierdo debido a un crecimiento pronunciado de dicha pared ventricular ${ }^{11}$. En otro estudio, en el que se analizaron nueve genes en 197 casos índice de $\mathrm{MCH}$ (incluidos 172 casos familiares y 25 aparentemente esporádicos), Richard, et al. ${ }^{107}$ encontraron variantes patogénicas en el $63 \%$ de los casos; de éstos, $42 \%$ se encontraba en la secuencia del gen MYBPC3 y $40 \%$ en $\mathrm{MYH}^{7}$ Ambos genes codifican a proteínas de filamentos gruesos y cabe señalar que este tipo de hallazgos se repite a través de diversas investigaciones ${ }^{108,109}$ en distintos grupos étnicos. Lo anterior convierte a los genes MYBPC3 y MYH7 como el principal objeto de estudio ante casos de $\mathrm{MCH}$ con antecedente de muerte súbita familiar o paro cardíaco no letal (Tabla 2).

\section{El gen MYBPC3 y su papel en la $\mathrm{MCH}$}

El gen MYBPC3 se localiza en el brazo corto del cromosoma 11, el cual codifica a la isoforma cardíaca de la proteína $C$ de unión a la miosina (MYBPC o MyBPC, myosin-binding protein C). Esta proteína se expresa sólo en el músculo cardíaco y se localiza de modo específico en las bandas A transversales del sarcómero, unida a las cadenas pesadas de miosina en los filamentos gruesos; por lo tanto, se la considera 
miembro del complejo tripartito junto con la actina y la miosina, y regula así la contracción cardíaca a través de sucesos de fosforilación ${ }^{101,110}$.

El mecanismo por el cual sus variantes patogénicas llevan al desarrollo de $\mathrm{MCH}$ aún es tema de debate, pero se han propuesto dos hipótesis. Una de ellas atribuye las manifestaciones clínicas a la haploinsuficiencia, con aumento de la sensibilidad al calcio ${ }^{111}$, mientras que la segunda favorece la hipótesis de un efecto tóxico de la proteína alterada ${ }^{112}$.

El estudio del gen MYBPC3 cobró interés a raíz del uso cada vez más frecuente para el diagnóstico de las tecnologías de biología molecular, las cuales han encontrado una relación consistente entre variantes patogénicas de este gen y el desarrollo de $\mathrm{MCH}^{113}$ y hasta el momento se han documentado más de 450 variantes patogénicas o probablemente patogénicas en la The Human Gene Mutation Database (HGMD; http://www. hgmd.cf.ac.uk).

El estudio molecular en casos de muerte súbita llevó a diversos investigadores a la vinculación con $\mathrm{MCH}$ debido a la presencia de variantes patogénicas en proteínas que intervienen en el funcionamiento del sarcómero. En este contexto, Cann, et al. ${ }^{49}$ realizaron un estudio de necropsia molecular en 96 casos de muerte súbita cardíaca e identificaron 50 casos con miocardiopatía, de los cuales 15 tuvieron $\mathrm{MCH}$ y de éstos tres presentaron variantes patogénicas en el gen MYBPC3 y tenían el antecedente de muerte súbita de origen cardíaco durante el ejercicio, inmediatamente después de realizar ejercicio o durante el sueño ${ }^{49}$. Las características clínicas de inicio tardío y alta frecuencia de antecedentes familiares de muerte súbita en los afectados se repiten a través de los múltiples estudios en distintas poblaciones alrededor del mundo ${ }^{53,113}$.

\section{El gen $M Y H 7$ y su papel en la $\mathrm{MCH}$}

El gen MYH7 se localiza en el brazo largo del cromosoma 14 , codifica a la isoforma $\beta$ de la cadena pesada de miosina (MYH o B-MHC, myosin heavy chain), proteína que se expresa en el músculo estriado de los mamíferos (de forma predominante en los ventrículos) y que forma parte de los filamentos gruesos del sarcómero ${ }^{114}$. MYH7 es el primer gen cuyas variantes patogénicas se relacionaron con $\mathrm{MCH}$ y cuyo efecto dominante negativo está bien descrito ${ }^{115,116}$.

Hasta la fecha se han documentado más de 350 variantes patogénicas en el gen MYH7 en HGMD, con fenotipos clínicos altamente variables, como es el estudio de cohorte de García-Castro, et al. ${ }^{108}$ en 120 pacientes no emparentados con $\mathrm{MCH}$. A los sujetos de este estudio se les realizó historia clínica y estudio molecular, y se identificaron 31 variantes patogénicas distintas en 32 pacientes, $8 \%$ de los cuales (10 pacientes) tenía alteración en el gen $M Y H 7$; la media de edad a la que se estableció el diagnóstico fue de 35 años y 3 de estos 10 pacientes (70\%) tenían el antecedente familiar de $\mathrm{MCH}^{108}$.

En cuanto al pronóstico de los portadores de variantes patogénicas del gen MYH7, Wang, et al. ${ }^{117}$ informaron en 2008 que las variantes patogénicas de este gen producen fenotipos más agresivos en comparación con aquellas que afectan al gen $M Y B P C 3$, con manifestaciones de inicio temprano (16 años) y alto riesgo de muerte súbita ${ }^{117}$.

Diversas investigaciones han tratado de establecer la correlación genotipo-fenotipo para facilitar el diagnóstico temprano en sujetos asintomáticos, pero esto ha sido difícil de establecer debido al gran número de variantes patogénicas identificadas en diversos genes (heterogeneidad de locus), a un posible efecto aditivo que modifique el fenotipo como en el caso de la edad de presentación y el pronóstico, a la gran variabilidad clínica de esta enfermedad y a la influencia del ambiente ${ }^{48}$.

\section{Variantes patogénicas o probablemente patogénicas localizadas en MYBPC3 y MYH7 en relación con muerte súbita}

Hoy en día existe una decena de variantes patogénicas o probablemente patogénicas relacionadas con muerte súbita en diferentes poblaciones alrededor del mundo; la mayor parte de ellas produce un desplazamiento del marco de lectura y, como consecuencia, la proteína sintetizada es trunca debido a un codón de paro prematuro. A continuación se describen de forma sintética algunas de ellas en el contexto de la muerte súbita.

\section{Variantes relacionadas con $\mathrm{MCH}$ y antecedente de muerte súbita para el gen MYBPC3}

p.Glu542GIn: Este cambio se ha descrito en siete casos índices no relacionados. En el plano molecular se ve afectado el último nucleótido de un sitio consenso para corte y empalme (splicing) y como efecto se obtiene una proteína corta de 486 aminoácidos ${ }^{118}$.

p.Cys719Arg: Este cambio se identificó en el año 2017 en un caso aparentemente esporádico de ascendencia 
china cuya causa de muerte era desconocida y en el informe de defunción se concluyó muerte súbita ${ }^{47}$.

p.Glu334Lys: Se identificó en un hombre de 48 años, de ascendencia coreana, diagnosticado por medios ecocardiográficos como una fenocopia del síndrome de Brugada al momento del diagnóstico de ingreso. Era un sobreviviente de un paro cardíaco no letal ${ }^{100}$.

p.Pro108Alafs*9: Se trata de una inserción de los nucleótidos GCTGGCCCCTGCC en la posición 29 del exón 3. La variante se identificó de modo inicial en 13 familias del sur de España, por lo que se estudió a 107 familiares y de ellos 39 tenían $\mathrm{MCH}$, con predominio del sexo masculino y con cinco casos de muerte súbita documentados y una mayor masa ventricular izquierda para los portadores de esta variante ${ }^{43}$. En la proteína, la longitud se reduce a 115 aminoácidos, ocho de los cuales son nuevos.

p.Gly1093Cys: Se analizaron 96 casos de necropsias de muerte súbita, en los cuales un probando falleció inmediatamente después de realizar ejercicio, motivo por el cual se solicitó prueba genética a 12 familiares, ocho de los cuales fueron positivos para este cambio y dos casos tenían síntomas cardíacos relacionados con $\mathrm{MCH}^{49}$.

p.Arg668His: El caso índice falleció en apariencia durante el sueño, motivo por el que se estudió a 27 familiares de los cuales 10 resultaron positivos para la variante y cuatro experimentaron síntomas relacionados con $\mathrm{MCH}^{49}$. La variante se había notificado ya con anterioridad ${ }^{119}$.

p.Arg502Trp: El caso índice falleció durante la realización de ejercicio, motivo por el cual se realizó estudio genético a cinco familiares, de los cuales tres fueron positivos, uno de ellos con síntomas relacionados con $\mathrm{MCH}^{49}$.

IVS5+5G $\rightarrow$ C: Esta variante corresponde a una sustitución de guanina por citocina cinco pares de bases corriente abajo 5' del sitio donador de corte y empalme en el intrón 5 del gen MYBPC3. Esta modificación ocasiona un cambio en el marco de lectura y añade 15 aminoácidos después de la posición 165, lo que causa al final una proteína trunca. Lin, et al. informan el caso de una familia en la que existe el antecedente de muerte súbita en el hermano del probando, quien falleció a la edad de 20 años. Tanto el padre como el probando son portadores clínicos de $\mathrm{MCH}^{120}$.

p.F305Pfs*27: Se trata de una deleción de dos timinas en el exón 11 que causa un codón de terminación y por lo tanto una proteína trunca. Calore, et al. estudiaron a una serie de 97 probandos de origen italiano con diagnóstico de $\mathrm{MCH}$, de los cuales 19 fueron portadores de la variante, más 45 portadores detectados por tamizaje en cascada. La penetrancia en esta serie de pacientes fue del $75 \%$; en ocho portadores hubo antecedente de muerte súbita, por lo cual el pronóstico es reservado para aquéllos con la variante después de la cuarta década de la vida ${ }^{52}$.

Lys1209Serfs²8: En combinación con p.Gly100Ser localizada en PRKAG2 (probable efecto aditivo), Zhao, et al. estudiaron a 18 pacientes con diagnóstico de $\mathrm{MCH}$, entre los cuales un probando presentó dos variantes y el antecedente de muerte súbita en la madre y abuela materna; el fenotipo del probando es consistente con hipertrofia grave e inicio temprano de $\mathrm{MCH}^{23}$.

\section{Variantes relacionadas con $\mathrm{MCH}$ y antecedente de muerte súbita para el gen MYH7}

p.Arg453Cys: En esta variante se observa un cambio de carga debido a un cambio de aminoácido, por lo que se ha informado un fenotipo más agresivo en comparación con Val606Met y Phe85Cys ${ }^{121,122}$. Ko, et al. estudiaron a un grupo de 20 individuos provenientes de una familia, todos ellos mayores de 16 años; en 11 casos se sospechó MCH clínica y en siete casos la enfermedad se confirmó por medio de ecocardiografía. Esta familia cuenta con el antecedente de tres casos de muerte súbita y dos con enfermedad cardíaca terminal ${ }^{108}$.

p.Arg1045Leu: En esta familia cuyo probando falleció durante el sueño se estudió a cuatro individuos en quienes se identificó a un familiar con datos clínicos de $\mathrm{MCH}^{49}$.

p.Arg719Trp: Anan, et al. realizaron un estudio genético en cuatro distintas familias con antecedente de $\mathrm{MCH}$ y por lo menos 22 casos de muerte súbita, lo que determinó un mayor riesgo de efectos adversos para los portadores de la variante ${ }^{123}$.

p.Asn391Thr: Feng, et al. estudiaron a una familia extensa de la etnia han (China) con diagnóstico de $\mathrm{MCH}$, la cual incluyó a tres generaciones y 22 individuos, de los cuales fallecieron tres con diagnóstico de $\mathrm{MCH}$; la edad de inicio de los síntomas de la enfermedad en su mayoría fue $<20$ años ${ }^{58}$.

p.Gly716Arg: Hwang, et al. estudiaron a una familia coreana compuesta por 32 miembros en cuatro generaciones. En esta familia existía el antecedente de cuatro individuos con muerte súbita a edad temprana; las pruebas genéticas demostraron que 13 individuos eran portadores de la variante ${ }^{124}$.

p.Arg403GIn: Marian, et al. estudiaron a una familia con siete casos positivos para esta variante. En esta 
familia, el inicio de los síntomas ocurrió en promedio a los 22 años; sin embargo, un individuo masculino de 10 años estaba asintomático. El análisis de sobrevida de Kaplan-Meier mostró que los portadores de la variante p.Arg403GIn tienen $11 \%$ de probabilidad de seguir vivos a los 60 años ${ }^{125}$. Esta variante se había notificado antes con alta incidencia para muerte súbita en otras tres familias ${ }^{126-128}$.

p.Arg453Cys: Esta variante se reconoció en el decenio de 1990 en una familia con 13 individuos afectados con $\mathrm{MCH}$ y el antecedente de seis casos de muerte súbita. De forma inicial, el tamizaje para detectar variantes se realizó con ayuda de estudios de protección de ribonucleasas y en el plano familiar se vinculó la enfermedad con la variante encontrada por medio de análisis de ligamiento; este cambio produce un cambio en la carga del aminoácido (-1) y se ha relacionado con una menor sobrevida ${ }^{128}$.

p.Glu848Gly: Esta sustitución de ácido glutámico por glicina da lugar a un efecto dominante negativo mediante la alteración de uniones proteína-proteína (MYH7-MYPC3) que llevan a la disfunción sistólica y riesgo aumentado de muerte súbita ${ }^{67}$.

p.Asn391Thr: Variante sin sentido hallada durante el estudio de una familia originaria de China con múltiples individuos afectados por $\mathrm{MCH}$ e historial de cuatro integrantes con muerte súbita ${ }^{58}$.

\section{Conclusión}

La $\mathrm{MCH}$ es una enfermedad altamente heterogénea debido al gran número de genes afectados e identificados como causales; además, la presencia de una o más mutaciones en proteínas integrantes del aparato contráctil del músculo cardíaco y al final la influencia ambiental dan origen a un fenotipo particular con curso clínico variable, en el cual la muerte súbita puede ser la primera manifestación. Entre los genes que codifican a proteínas integrantes del aparato contráctil del músculo cardíaco, los genes de filamentos gruesos MYBPC3 y $M Y H 7$ son los identificados con más frecuencia como causales en las publicaciones médicas mundiales, tomadas en cuenta todas las poblaciones, con más de 350 variantes patogénicas o probablemente patogénicas notificadas en cada gen.

\section{Conflicto de intereses}

Ninguno.

\section{Financiamiento}

La presente investigación no ha recibido ninguna beca específica de agencias de los sectores público, comercial, o sin ánimo de lucro.

\section{Responsabilidades éticas}

Protección de personas y animales. Los autores declaran que para esta investigación no se han realizado experimentos en seres humanos ni en animales.

Confidencialidad de los datos. Los autores declaran que en este artículo no aparecen datos de pacientes.

Derecho a la privacidad y consentimiento informado. Los autores declaran que en este artículo no aparecen datos de pacientes.

\section{Agradecimientos}

Los autores agradecen la colaboración de Leonardo Olguín Landa en la elaboración de la figura 1.

\section{Bibliografía}

1. Elliott $P$, Andersson $B$, Arbustini E, Bilinska Z, Cecchi F, Charron $P$, et al. Classification of the cardiomyopathies: a position statement from the European Society Of Cardiology Working Group on Myocardial and Pericardial Diseases. Eur Heart J 2008(2);29:270-6.

2. Maron BJ, Gardin JM, Flack JM, Gidding SS, Kurosaki TT, Bild DE. Prevalence of hypertrophic cardiomyopathy in a general population of young adults. Echocardiographic analysis of 4111 subjects in the CARDIA Study. Coronary Artery Risk Development in (Young) Adults. Circulation 1995(4);92:785-9.

3. Bagnall RD, Weintraub RG, Ingles J, Duflou J, Yeates L, Lam L, et al A prospective study of sudden cardiac death among children and young adults. N Engl J Med 2016(25);374:2441-52.

4. Michels M, Soliman OI, Phefferkorn J, Hoedemaekers YM, Kofflard MJ, Dooijes D, et al. Disease penetrance and risk stratification for sudden cardiac death in asymptomatic hypertrophic cardiomyopathy mutation carriers. Eur Heart J 2009(21);30:2593-8.

5. McKenna WJ, Spirito P, Desnos M, Dubourg O, Komajda M. Experience from clinical genetics in hypertrophic cardiomyopathy: proposal for new diagnostic criteria in adult members of affected families. Heart 1997(2);77:130-2.

6. Brugada J. Sudden death in hypertrophic myocardiopathy. Rev Esp Cardiol 1998(12);51:991-6.

7. Sirker A, Shah AM. Biochemistry and physiology of cardiac muscle. Medicine 2010(7);38:340-3.

8. Tian T, Liu Y, Zhou X, Song L. Progress in the molecular genetics of hypertrophic cardiomyopathy: a mini-review. Gerontology 2013(3);59:199-205.

9. Crilley JG, Boehm EA, Blair E, Rajagopalan B, Blamire AM, Styles P, et al. Hypertrophic cardiomyopathy due to sarcomeric gene mutations is characterized by impaired energy metabolism irrespective of the degree of hypertrophy. J Am Coll Cardiol 2003(10);41:1776-82.

10. Ashrafian $\mathrm{H}$, Redwood $\mathrm{C}$, Blair $\mathrm{E}$, Watkins $\mathrm{H}$. Hypertrophic cardiomyopathy: a paradigm for myocardial energy depletion. Trends Genet 2003(5);19:263-8.

11. Coppini R, Ho CY, Ashley E, Day S, Ferrantini C, Girolami F, et al. Clinical phenotype and outcome of hypertrophic cardiomyopathy associated with thin-filament gene mutations. J Am Coll Cardiol 2014(24);64:2589-600.

12. Monserrat L, Hermida-Prieto M, Fernández X, Rodríguez I, Dumont $C$, Cazón L, et al. Mutation in the alpha-cardiac actin gene associated with apical hypertrophic cardiomyopathy, left ventricular non-compaction, and septal defects. Eur Heart J 2007(16);28:1953-61.

13. Gómez J, Lorca R, Reguero JR, Morís C, Martín M, Tranche S, et al. Screening of the filamin $\mathrm{c}$ gene in a large cohort of hypertrophic cardiomyopathy patients. Circ Cardiovasc Genet 2017(2);10:e001584.

14. Ingles J, Burns C, Bagnall RD, Lam L, Yeates L, Sarina T, et al. Nonfa- 
milial hypertrophic cardiomyopathy: prevalence, natural history, and clinical implications. Circ Cardiovasc Genet 2017(2);10:e001620.

15. Frustaci A, De Luca A, Guida V, Biagini T, Mazza T, Gaudio C, et al. Novel $\alpha$-actin gene mutation $\mathrm{p}$.(ala21val) causing familial hypertrophic cardiomyopathy, myocardial noncompaction, and transmural crypts. Clinical-pathologic correlation. J Am Heart Assoc 2018(4);7:e008068.

16. Yang QL, Bian YY, Wang B, Zuo L, Zhou MY, Shao H, et al. Novel phenotype-genotype correlations of hypertrophic cardiomyopathy caused by mutation in $\alpha$-actin and myosin-binding protein genes in three unrelated Chinese families. J Cardiol 2018(18); S0914-5087:30328-9.

17. Nagyova E, Radvanszky J, Hyblova M, Simovicova V, Goncalvesova E, Asselbergs FW, et al. Targeted next-generation sequencing in Slovak cardiomyopathy patients. Bratisl Lek Listy 2019(1;120):46-51.

18. Chung WK, Kitner C, Maron BJ. Novel frameshift mutation in troponin $C$ (TNNC1) associated with hypertrophic cardiomyopathy and sudden death. Cardiol Young 2011(3);21:345-8.

19. Van Driest SL, Ellsworth EG, Ommen SR, Tajik AJ, Gersh BJ, Ackerman MJ. Prevalence and spectrum of thin filament mutations in an outpatient referral population with hypertrophic cardiomyopathy. Circulation 2003(4):108:445-51.

20. Rani DS, Nallari $P$, Priyamvada $S$, Narasimhan $C$, Singh L, Thangaraj $K$ High prevalence of arginine to glutamine substitution at 98, 141 and 162 positions in troponin I (TNNI3) associated with hypertrophic cardiomyopathy among Indians. BMC Med Genet 2012;13:69.

21. Zigova M, Bernasovska J, Boronova I, Mydlarova Blascakova M, Kmec J. Finding the candidate sequence variants for diagnosis of hypertrophic cardiomyopathy in East Slovak patients. J Clin Lab Anal 2018(3);32:e22303

22. Mademont-Soler I, Mates J, Yotti R, Espinosa MA, Pérez-Serra A, Fernandez-Avila Al, et al. Additional value of screening for minor genes and copy number variants in hypertrophic cardiomyopathy. PLoS One 2017(8):12:e0181465

23. Zhao $Y$, Feng $Y$, Ding $X$, Dong $S$, Zhang $H$, Ding J, et al. Identification of a novel hypertrophic cardiomyopathy-associated mutation using targeted next-generation sequencing. Int J Mol Med 2017(1);40:121-9.

24. Sohn DW, Kim HK, Kim YJ, Oh S, Seong MW, Park SS. Cardiomyopathies with mixed and inapparent morphological features in cardiac troponin i3 mutation. Korean Circ J 2017(3);47:413-7.

25. Anan R, Shono H, Kisanuki A, Arima S, Nakao S, Tanaka H. Patients with familial hypertrophic cardiomyopathy caused by a Phe110lle missense mutation in the cardiac troponin $\mathrm{T}$ gene have variable cardiac morphologies and a favorable prognosis. Circulation 1998(5); 98:391-7.

26. Mendes de Almeida R, Tavares J, Martins S, Carvalho T, Enguita FJ, Brito $D$, et al. Whole gene sequencing identifies deep-intronic variants with potential functional impact in patients with hypertrophic cardiomyopathy. PLoS One 2017(8);12:e0182946.

27. Biswas A, Das S, Kapoor M, Shamsudheen KV, Jayarajan R, Verma A, et al. Familial hypertrophic cardiomyopathy - identification of cause and risk stratification through exome sequencing. Gene 2018:660:151-156.

28. Karibe A, Tobacman LS, Strand J, Butters C, Back N, Bachinski LL, et al. Hypertrophic cardiomyopathy caused by a novel alpha-tropomyosin mutation (V95A) is associated with mild cardiac phenotype, abnormal calcium binding to troponin, abnormal myosin cycling, and poor prognosis. Circulation 2001(1);103:65-71.

29. Renaudin P, Janin A, Millat G, Chevalier P. A novel missense mutation p.gly162glu of the gene myl2 involved in hypertrophic cardiomyopathy: a pedigree analysis of a proband. Mol Diagn Ther 2018(2);22:219-223.

30. Weissler-Snir A, Hindieh W, Gruner C, Fourey D, Appelbaum E, Rowin E, et al. Lack of phenotypic differences by cardiovascular magnetic resonance imaging in myh7 ( $\beta$-myosin heavy chain)- versus mybpc3 (myosin-binding protein c)-related hypertrophic cardiomyopathy. Circ Cardiovasc Imaging 2017(2);10:e005311.

31. McTaggart DR, Ogden KJ, Marathe JA. A long term follow-up study of carriers of hypertrophic cardiomyopathy mutations. Heart Lung Circ 2017(1);26:18-24

32. Chida A, Inai K, Sato H, Shimada E, Nishizawa T, Shimada M. Prognostic predictive value of gene mutations in Japanese patients with hypertrophic cardiomyopathy. Heart Vessels 2017(6);32:700-7.

33. Cirino AL, Lakdawala NK, McDonough B, Conner L, Adler D, Weinfeld M, et al. A comparison of whole genome sequencing to multigene panel testing in hypertrophic cardiomyopathy patients. Circ Cardiovasc Genet 2017(5);10:e001768.

34. Kissopoulou A, Trinks C, Green A, Karlsson J, Jonasson J, Gunnarsson C. Homozygous missense MYBPC3 Pro873His mutation associated with increased risk for heart failure development in hypertrophic cardiomyopathy. ESC Heart Fail 2018(4);5:716-23.

35. Lorca R, Gómez J, Martín M, Cabanillas R, Calvo J, León V, et al. Insights into hypertrophic cardiomyopathy evaluation through follow-up of a founder pathogenic variant. Rev Esp Cardiol (Engl Ed) 2019(2);72:138-44

36. Nomura A, Tada $\mathrm{H}$, Teramoto $\mathrm{R}$, Konno $\mathrm{T}$, Hodatsu A, Won $\mathrm{HH}$, et al. Whole exome sequencing combined with integrated variant annotation prediction identifies a causative myosin essential light chain variant in hypertrophic cardiomyopathy. J Cardiol 2016(2);67:133-9.
37. Zhou N, Qin S, Liu Y, Tang L, Zhao W, Pan C, et al. Whole-exome sequencing identifies rare compound heterozygous mutations in the MYBPC3 gene associated with severe familial hypertrophic cardiomyopathy. Eur J Med Genet 2018(17)S1769-7212:30728-0.

38. Hallıoğlu Kılınç O, Giray D1, Bişgin A, Tuğ Bozdoğan S, Karpuz D. Familial hypertrophic cardiomyopathy: a case with a new mutation in the MYBPC3 gene. Turk Kardiyol Dern Ars 2017(5):45:450-3.

39. McNamara JW, Li A, Lal S, Bos J, Harris S, van der Velden J. MYBPC3 mutations are associated with a reduced super-relaxed state in patients with hypertrophic cardiomyopathy. PLoS One 2017(6);12:e0180064.

40. Szabadosova V, Boronova I, Ferenc P, Tothova I, Bernasovska J, Zigova M, et al. Analysis of selected genes associated with cardiomyopathy by next-generation sequencing. J Clin Lab Anal 2018(2;32).

41. Rafael JF, Cruz FEDS Filho, Carvalho ACC, Gottlieb I, Cazelli JG, Siciliano AP, et al. Myosin-binding protein c compound heterozygous variant effect on the phenotypic expression of hypertrophic cardiomyopathy. Arq Bras Cardiol 2017(4);108:354-60.

42. Zhao B, Wang S, Chen J, Ji Y, Wang J, Tian X, et al. Echocardiographic characterization of hypertrophic cardiomyopathy in Chinese patients with myosin-binding protein C3 mutations. Exp Ther Med 2017(3)13;995-1002.

43. Sabater-Molina M, Saura D, García-Molina Sáez E, González-Carrillo J, Polo L, Pérez-Sánchez I, et al. A novel founder mutation in mybpc3: phenotypic comparison with the most prevalent mybpc3 mutation in Spain. Rev Esp Cardiol (Engl Ed) 2017(2);70:105-14.

44. Cen X, Zheng J, Hu X, Qu B. Severe apical hypertrophic cardiomyopathy with Ser 236 Gly mutation in MYBPC3: A three-year follow-up investigation. Hellenic J Cardiol 2017(5);58:366-8.

45. Bottillo I, D'Angelantonio D, Caputo V Paiardini A, Lipari M De Bernardo C, et al. Molecular analysis of sarcomeric and non-sarcomeric genes in patients with hypertrophic cardiomyopathy. Gene 2016(2); 577:227-35

46. Mathew J, Zahavich L, Lafreniere-Roula M, Wilson J, George K, Benson L, et al. Utility of genetics for risk stratification in pediatric hypertrophic cardiomyopathy. Clin Genet 2018(2);93:310-9.

47. Xu CC, Bai YZ, Xu XS, Lü GL, Lai XP, Chen R. Gene analysis for the sudden death of hypertrophic cardiomyopathy by whole exome sequencing. Fa Yi Xue Za Zhi 2017(4);33:339-43.

48. Zhang X, Xie J, Zhu S, Chen Y, Wang L, Xu B. Next-generation sequencing identifies pathogenic and modifier mutations in a consanguineous Chinese family with hypertrophic cardiomyopathy. Medicine (Baltimore) 2017(24);96:e7010.

49. Cann F, Corbett M, O'Sullivan D, Tennant S, Hailey H, Grieve JH, et al. Phenotype-driven molecular autopsy for sudden cardiac death. Clin Genet 2017(1);91:22-9

50. Mak CM, Chen SP, Mok NS, Siu WK, Lee HH, Ching CK, et al. Genetic basis of channelopathies and cardiomyopathies in Hong Kong Chinese patients: a 10-year regional laboratory experience. Hong Kong Med J 2018(4);24:340-9.

51. Guo $X$, Fan $C$, Tian L, Liu Y, Wang $H$, Zhao $S$, et al. The clinical features, outcomes and genetic characteristics of hypertrophic cardiomyopathy patients with severe right ventricular hypertrophy. PLoS One 2017(3); 12:e0174118.

52. De Bortoli M, Calore C, Lorenzon A, Calore M, Poloni G, Mazzotti E, et al. Co-inheritance of mutations associated with arrhythmogenic cardiomyopathy and hypertrophic cardiomyopathy. Eur J Hum Genet 2017(10);25:1165-9.

53. Teirlinck CH, Senni F, Malti RE, Majoor-Krakauer D, Fellmann F, Millat G, et al. A human MYBPC3 mutation appearing about 10 centuries ago results in a hypertrophic cardiomyopathy with delayed onset, moderate evolution but with a risk of sudden death. BMC Med Genet 2012:13:105.

54. Page SP, Kounas S, Syrris P, Christiansen M, Frank-Hansen R, Andersen PS, et al. Cardiac myosin binding protein-C mutations in families with hypertrophic cardiomyopathy: disease expression in relation to age, gender, and long term outcome. Circ Cardiovasc Genet 2012(2);5: $156-66$.

55. Ross SB, Bagnall RD, Ingles J, Van Tintelen JP, Semsarian C. Burden of recurrent and ancestral mutations in families with hypertrophic cardiomyopathy. Circ Cardiovasc Genet 2017(3);10:e001671.

56. Carniel E, Taylor MR, Sinagra G, Di Lenarda A, Ku L, Fain PR, et al Alpha-myosin heavy chain: a sarcomeric gene associated with dilated and hypertrophic phenotypes of cardiomyopathy. Circulation 2005(1); 112:54-9.

57. Arai S, Matsuoka R, Hirayama K, Sakurai H, Tamura M, Ozawa T, et al. Missense mutation of the beta-cardiac myosin heavy-chain gene in hypertrophic cardiomyopathy. Am J Med Genet 1995(3);58:267-76.

58. Feng $X$, He T, Wang JG, Zhao P. Asn391Thr mutation of $\beta$-myosin heavy chain in a hypertrophic cardiomyopathy family. Int Heart J 2018(3); 59:596-600.

59. Montag J, Syring M, Rose J, Weber AL, Ernstberger P, Mayer AK, et al. Intrinsic MYH7 expression regulation contributes to tissue level allelic imbalance in hypertrophic cardiomyopathy. J Muscle Res Cell Motil 2017(3-4);38:291-302.

60. Wang B, Guo RQ, Wang J, Yang F, Zuo L, Liu Y, et al. The cumulative 
effects of the myh7-v878a and cacna1c-a1594v mutations in a chinese family with hypertrophic cardiomyopathy. Cardiology 2017(4);138:228-37.

61. Wang C, Hata Y, Hirono K, Takasaki A, Ozawa SW, Nakaoka H, et al A wide and specific spectrum of genetic variants and genotype-phenotype correlations revealed by next-generation sequencing in patients with left ventricular noncompaction. J Am Heart Assoc 2017(9);6:e006210.

62. Wang B, Guo R, Zuo L, Shao H, Liu Y, Wang Y, et al. Analysis of genotype and phenotype correlation of MYH7-V878A mutation among ethnic Han Chinese pedigrees affected with hypertrophic cardiomyopathy. Zhonghua Yi Xue Yi Chuan Xue Za Zhi 2017(4);34:514-8.

63. Gawor M, Bilińska ZT, Franaszczyk M, Michalak E, Rafał P, Grzybowski J. A new missense mutation, p.Arg719Leu, of the beta-myosin heavy chain gene in a patient with familial hypertrophic cardiomyopathy. Minerva Cardioangiol 2017(1);65:96-102.

64. Montag J, Kowalski K, Makul M, Ernstberger P, Radocaj A, Beck J, et al. Burst-like transcription of mutant and wildtype myh7-alleles as possible origin of cell-to-cell contractile imbalance in hypertrophic cardiomyopathy. Front Physiol 2018:359:9.

65. Wang J, Wan K, Sun J, Li W, Liu H, Han Y3, et al. Phenotypic diversity identified by cardiac magnetic resonance in a large hypertrophic cardiom yopathy family with a single MYH7 mutation. Sci Rep 2018(1):8:973.

66. Guo X, Fan C, Wang Y, Wang M, Cai C, Yang Y, et al. Genetic anticipation in a special form of hypertrophic cardiomyopathy with sudden cardiac death in a family with 74 members across 5 generations. Medicine (Baltimore) 2017(11);96:e6249.

67. Yang KC, Breitbart A, De Lange W, Hofsteen P, Futakuchi-Tsuchida A, et al. Novel adult-onset systolic cardiomyopathy due to myh7 e848g mutation in patient-derived induced pluripotent stem cells. JACC Basic TransI Sci 2018(6);3:728-40.

68. Lu C, Wu W, Liu F, Yang K, Li J, Liu Y, et al. Molecular analysis of inherited cardiomyopathy using next generation semiconductor sequencing technologies. J Transl Med 2018(1):16:241.

69. Goel N, Huddleston CB, Fiore AC. A novel mutation of the MYH7 gene in a patient with hypertrophic cardiomyopathy. Turk J Pediatr 2018(3); 60:315-8

70. Kabaeva ZT, Perrot A, Wolter B, Dietz R, Cardim N, Correia JM, et al. Systematic analysis of the regulatory and essential myosin light chain genes: genetic variants and mutations in hypertrophic cardiomyopathy. Eur J Hum Genet 2002(11):10:741-8.

71. Flavigny J, Richard P, Isnard R, Carrier L, Charron P, Bonne G, et al. Identification of two novel mutations in the ventricular regulatory myosin light chain gene (MYL2) associated with familial and classical forms of hypertrophic cardiomyopathy. J Mol Med (Berl) 1998(3-4);76:208-14.

72. Li L, Bainbridge MN, Tan Y, Willerson JT, Marian AJ. A potential oligogenic etiology of hypertrophic cardiomyopathy: a classic single-gene disorder. Circ Res 2017(7);120:1084-90.

73. Jay A, Chikarmane R, Poulik J, Misra VK. Infantile hypertrophic cardiomyopathy associated with a novel MYL3 mutation. Cardiology 2013(4); 124:248-51.

74. Olson TM, Karst ML, Whitby FG, Driscoll DJ. Myosin light chain mutation causes autosomal recessive cardiomyopathy with mid-cavitary hypertrophy and restrictive physiology. Circulation 2002(20):105:2337-40.

75. Arad M, Penas-Lado M, Monserrat L, Maron BJ, Sherrid M, Ho CY, et al. Gene mutations in apical hypertrophic cardiomyopathy. Circulation 2005(18);112:2805-11

76. Girolami F, lascone M, Tomberli B, Bardi S, Benelli M, Marseglia G, et al. Novel $\alpha$-actinin 2 variant associated with familial hypertrophic cardiomyopathy and juvenile atrial arrhythmias: a massively parallel sequencing study. Circ Cardiovasc Genet 2014(6):7:741-50.

77. Chiu C, Bagnall RD, Ingles J, Yeates L, Kennerson M, Donald JA, et al. Mutations in alpha-actinin-2 cause hypertrophic cardiomyopathy: a genome-wide analysis. J Am Coll Cardiol 2010(11);55:1127-35

78. Geier C, Gehmlich K, Ehler E, Hassfeld S, Perrot A, Hayess K, et al Beyond the sarcomere: CSRP3 mutations cause hypertrophic cardiomyopathy. Hum Mol Genet 2008(18)17:2753-65.

79. Janin A, Bessière F, Chauveau S, Chevalier P, Millat G. First identification of homozygous truncating CSRP3 variants in two unrelated cases with hypertrophic cardiomyopathy. Gene 2018;676:110-116.

80. Theis JL, Bos JM, Bartleson VB, Will ML, Binder J, Vatta M, et al. Echocardiographic-determined septal morphology in Z-disc hypertrophic cardiomyopathy. Biochem Biophys Res Commun 2006(4);351:896-902.

81. Osio A, Tan L, Chen SN, Lombardi R, Nagueh SF, Shete S, et al. Myozenin 2 is a novel gene for human hypertrophic cardiomyopathy. Circ Res 2007(6);100:766-8.

82. Purevjav E, Arimura T, Augustin S, Huby AC, Takagi K, Nunoda S, et al. Molecular basis for clinical heterogeneity in inherited cardiomyopathies due to myopalladin mutations. Hum Mol Genet 2012(9);21:2039-53.

83. Wang H, Li Z, Wang J, Sun K, Cui Q, Song L, et al. Mutations in NEXN, a Z-disc gene, are associated with hypertrophic cardiomyopathy. Am J Hum Genet 2010(5);87:687-93.

84. Hayashi T, Arimura T, Itoh-Satoh M, Ueda K, Hohda S, Inagaki N, et al. Tcap gene mutations in hypertrophic cardiomyopathy and dilated cardiomyopathy. J Am Coll Cardiol 2004(11);44:2192-201.
85. Campuzano O, Sánchez-Molero O, Mademont-Soler I, Riuró H, Allegue C, Coll M, et al. Rare titin (TTN) variants in diseases associated with sudden cardiac death. Int J Mol Sci 2015(10);16:25773-87.

86. Vasile VC, Will ML, Ommen SR, Edwards WD, Olson TM, Ackerman MJ. Identification of a metavinculin missense mutation, R975W, associated with both hypertrophic and dilated cardiomyopathy. Mol Genet Metab 2006(2);87:169-74.

87. Chiu C, Tebo M, Ingles J, Yeates L, Arthur JW, Lind JM, et al. Genetic screening of calcium regulation genes in familial hypertrophic cardiomyopathy. J Mol Cell Cardiol 2007(3);43:337-43.

88. Landstrom AP, Kellen CA, Dixit SS, van Oort RJ, Garbino A, Weisleder N, et al. Junctophilin-2 expression silencing causes cardiocyte hypertrophy and abnormal intracellular calcium-handling. Circ Heart Fail 2011(2);4: 214-23.

89. Vanninen SUM, Leivo K, Seppälä EH, Aalto-Setälä K, Pitkänen O, Suursalmi $\mathrm{P}$, et al. Heterozygous junctophilin-2 (JPH2) p.(Thr161Lys) is a monogenic cause for HCM with heart failure. PLoS One 2018(9); 13:e0203422.

90. Minamisawa S, Sato $Y$, Tatsuguchi $Y$, Fujino $T$, Imamura $S$, Uetsuka $Y$, et al. Mutation of the phospholamban promoter associated with hypertrophic cardiomyopathy. Biochem Biophys Res Commun 2003(1):304:1-4.

91. Hayashi T, Arimura T, Ueda K, Shibata H, Hohda S, Takahashi M, et al. Identification and functional analysis of a caveolin-3 mutation associated with familial hypertrophic cardiomyopathy. Biochem Biophys Res Commun 2004(1):313:178-84

92. Stull JT, Kamm KE, Vandenboom R. Myosin light chain kinase and the role of myosin light chain phosphorylation in skeletal muscle. Arch Biochem Biophys 2011(2);510:120-8.

93. Banankhah P, Fishbein GA, Dota A, Ardehali R. Cardiac manifestations of PRKAG2 mutation. BMC Med Genet 2018(1)19:1.

94. Torok RD, Austin SL, Phornphutkul C, Rotondo KM, Bali D2, Tatum GH, et al. PRKAG2 mutations presenting in infancy. J Inherit Metab Dis 2017(6);40:823-30

95. Xu Y, Gray A, Hardie DG, Uzun A, Shaw S, Padbury J, et al. A novel, de novo mutation in the PRKAG2 gene: infantile-onset phenotype and the signaling pathway involved. Am J Physiol Heart Circ Physiol 2017(2);313:H283-92.

96. Yang KQ, Lu CX, Zhang Y, Yang YK, Li JC, Lan T, et al. A novel PRKAG2 mutation in a Chinese family with cardiac hypertrophy and ventricular pre-excitation. Sci Rep 2017(1);7:2407.

97. Kelly BP, Russell MW, Hennessy JR, Ensing GJ. Severe hypertrophic cardiomyopathy in an infant with a novel PRKAG2 gene mutation: potential differences between infantile and adult onset presentation. Pediatr Cardiol 2009(8)30:1176-9.

98. Laforêt P, Richard P, Said MA, Romero NB, Lacene E, Leroy JP, et al. A new mutation in PRKAG2 gene causing hypertrophic cardiomyopathy with conduction system disease and muscular glycogenosis. Neuromuscul Disord 2006(3);16:178-82.

99. Jongbloed RJ, Marcelis CL, Doevendans PA, Schmeitz-Mulkens JM, Van Dockum WG, Geraedts JP, et al. Variable clinical manifestation of a novel missense mutation in the alpha-tropomyosin (TPM1) gene in familial hypertrophic cardiomyopathy. J Am Coll Cardiol 2003(6):41:981-6.

100. Song JS, Kang JS, Kim YE, Park SJ, Park KM, Huh J, et al. Identification of pathogenic variants in genes related to channelopathy and cardiomyopathy in Korean sudden cardiac arrest survivors. J Hum Genet 2017(6); 62:615-20.

101. Wang Y, Wang Z, Yang Q, Zou Y, Zhang H, Yan C, et al. Autosomal recessive transmission of MYBPC 3 mutation results in malignant phenotype of hypertrophic cardiomyopathy. PLoS One 2013(6):8:e67087.

102. Calore C, De Bortoli M, Romualdi C, Lorenzon A, Angelini A, Basso C, et al. A founder MYBPC3 mutation results in HCM with a high risk of sudden death after the fourth decade of life. J Med Genet 2015(5); 52:338-47.

103. Fourey D, Care M, Siminovitch KA, Weissler-Snir A, Hindieh W, Chan RH, et al. Prevalence and clinical implication of double mutations in hypertrophic cardiomyopathy: revisiting the gene-dose effect. Circ Cardiovasc Genet 2017(2);10:e001685.

104. Guo Q, Xu Y, Wang X, Guo Y, Xu R, Sun K, Chen S. Exome sequencing identifies a novel MYH7 p.G407C mutation responsible for familial hypertrophic cardiomyopathy. DNA Cell Biol 2014(10):44:699-704.

105. Ko YL, Chen JJ, Tang TK, Cheng JJ, Lin SY, Liou YC, et al. Malignant familial hypertrophic cardiomyopathy in a family with a $453 \mathrm{Arg}-->$ Cys mutation in the beta-myosin heavy chain gene: coexistence of sudden death and end-stage heart failure. Hum Genet 1996(5);97:585-90.

106. Andersen PS, Hedley PL, Page SP, Syrris P, Moolman-Smook JC, McKenna WJ, et al. A novel Myosin essential light chain mutation causes hypertrophic cardiomyopathy with late onset and low expressivity. Biochem Res Int 2012;2012:685108.

107. Richard P, Charron P, Carrier L, Ledeuil C, Cheav T, Pichereau C, et al. Hypertrophic cardiomyopathy: distribution of disease genes, spectrum of mutations, and implications for a molecular diagnosis strategy. Circulation 2003(17); 107:2227-32.

108. García-Castro M, Coto E, Reguero JR, Berrazueta JR, Álvarez V, 
Alonso $\mathrm{B}$, et al. Espectro mutacional de los genes sarcoméricos $\mathrm{MYH7}$, MYBPC3, TNNT2, TNNI3 y TPM1 en pacientes con miocardiopatía hipertrófica. Rev Esp Cardiol 2009(1)62:48-56.

109. Olivotto I, Girolami F, Ackerman MJ, Nistri S, Bos JM, Zachara E et al. Myofilament protein gene mutation screening and outcome of patients with hypertrophic cardiomyopathy. Mayo Clin Proc 2008(6) 83:630-8.

110. Previs MJ, Beck Previs S, Gulick J, Robbins J, Warshaw DM. Molecular mechanics of cardiac myosin-binding protein $C$ in native thick filaments. Science 2012(6099);337:1215-8.

111. Gaffin RD, Peña JR, Alves MS, Dias FA, Chowdhury SA, Heinrich LS, et al. Long-term rescue of a familial hypertrophic cardiomyopathy caused by a mutation in the thin filament protein, tropomyosin, via modulation of a calcium cycling protein. J Mol Cell Cardiol 2011(5);51:812-20.

112. Knöll R. Myosin binding protein C: implications for signal-transduction. J Muscle Res Cell Motil 2012(1);33:31-42.

113. Tanjore RR, Rangaraju A, Kerkar PG, Calambur N, Nallari P. MYBPC3 gene variations in hypertrophic cardiomyopathy patients in India. Can J Cardiol 2008(2);24:127-30

114. Warkman AS, Whitman SA, Miller MK, Garriock RJ, Schwach CM Gregorio CC, et al. Developmental expression and cardiac transcriptional regulation of Myh7b, a third myosin heavy chain in the vertebrate heart. Cytoskeleton (Hoboken) 2012(5);69:324-35.

115. Cuda G, Fananapazir L, Zhu WS, Sellers JR, Epstein ND. Skeletal muscle expression and abnormal function of beta-myosin in hypertrophic cardiomyopathy. J Clin Invest 1993(6);91:2861-5.

116. Lankford EB, Epstein ND, Fananapazir L, Sweeney HL. Abnormal contractile properties of muscle fibers expressing beta-myosin heavy chain gene mutations in patients with hypertrophic cardiomyopathy. J Clin Invest 1995(3);95:1409-14.

117. Wang S, Zou Y, Fu C, Xu X, Wang J, Song L, et al. Worse prognosis with gene mutations of beta-myosin heavy chain than myosin-binding protein $\mathrm{C}$ in Chinese patients with hypertrophic cardiomyopathy. Clin Cardiol 2008(3);31:114-8.

118. Carrier L, Bonne G, Bährend E, Yu B, Richard P, Niel F, et al. Organization and sequence of human cardiac myosin binding protein $C$ gene (MYBPC3) and identification of mutations predicted to produce truncated proteins in familial hypertrophic cardiomyopathy. Circ Res 1997(3);80:427-34.

119. Harris SP, Lyons RG, Bezold KL. In the thick of it: HCM-causing mutations in myosin binding proteins of the thick filament. Circ Res 2011(6); 108:751-64.

120. Lin J, Zheng DD, Tao Q, Yang JH, Jiang WP, Yang XJ, et al. Two novel mutations of the MYBPC3 gene identified in Chinese families with hypertrophic cardiomyopathy. Can J Cardiol 2010(10);26:518-22.

121. Epstein ND, Fananapazir L, Lin HJ, Mulvihill J, White R, Lalouel JM,et al. Evidence of genetic heterogeneity in five kindreds with familial hypertrophic cardiomyopathy. Circulation 1992(2);85:635-47.

122. Frisso G, Limongelli G, Pacileo G, Del Giudice A, Forgione L, Calabrò $P$ et al. A child cohort study from southern Italy enlarges the genetic spectrum of hyper-trophic cardiomyopathy. Clin Genet 2009(1);76:91-101.

123. Anan R, Greve G, Thierfelder L, Watkins H, McKenna WJ, Solomon S, et al. Prognostic implications of novel beta cardiac myosin heavy chain gene mutations that cause familial hypertrophic cardiomyopathy. J Clin Invest 1994(1):93:280-5

124. Hwang TH, Lee WH, Kimura A, Satoh M, Nakamura T, Kim MK, et al. Early expression of a malignant phenotype of familial hypertrophic cardiomyopathy associated with a Gly716Arg myosin heavy chain mutation in a Korean family. Am J Cardiol 1998(12);82:1509-13.

125. Marian AJ, Mares A Jr, Kelly DP, Yu QT, Abchee AB, Hill R, et al. Sudden cardiac death in hypertrophic cardiomyopathy. Variability in phenotypic expression of beta-myosin heavy chain mutations. Eur Heart $J$ 1995(3);16:368-76

126. Epstein ND, Cohn GM, Cyran F, Fananapazir L. Differences in clinical expression of hypertrophic cardiomyopathy associated with two distinct mutations in the beta-myosin heavy chain gene. A 908Leu-Val mutation and a 403Arg-GIn mutation. Circulation 1992(2);86:345-52.

127. Marian AJ, Yu QT, Mares A Jr, Hill R, Roberts R, Perryman M. Detection of a new mutation in the beta-myosin heavy chain gene in an individual with hypertrophic cardiomyopathy. J Clin Invest 1992(6);90:2156-65.

128. Watkins H, Rosenzweig A, Hwang DS, Levi T, McKenna W, Seidman CE, et al. Characteristics and prognostic implications of myosin missense mutations in familial hypertrophic cardiomyopathy. N Engl J Med 1992(17);326:1108-14. 